\title{
Value of information from condition inspection for a gamma degradation process
}

\author{
William Fauriat \\ Chair System Science and the Energy Challenge, Fondation EDF \\ CentraleSupélec, Université Paris-Saclay \\ Gif-sur-Yvette, France \\ *william.fauriat@centralesuperlec.fr \\ * Corresponding author
}

\author{
Enrico Zio ${ }^{1,2}$ \\ ${ }^{1}$ Energy Depertment \\ Politecnico di Milano \\ Milan, Italy \\ ${ }^{2}$ Center for research on Risks and Crises $(C R C)$, \\ MINES ParisTech, PSL University, \\ Sophia Antipolis, France \\ enrico.zio@polimi.it
}

\begin{abstract}
For components subject to continuous degradation appropriate maintenance decisions are needed for optimal operation in the presence of uncertainty regarding failure occurrence. Sometimes, uncertainty can be reduced through the collection of additional information on components' state. Value of Information (VoI) may be used to assess the impact of uncertainty reduction on the outcome of health management decisions. It is applied here in the context of a continuous degradation, modeled through a gamma process.
\end{abstract}

Keywords-Prognostics and Health Management, ConditionBased Maintenance, maintenance optimization, decision under uncertainty, Value of Information, gamma degradation process, preposterior analysis

\section{INTRODUCTION}

Components of mechanical and industrial systems are subject to degradation which can lead to failures with undesired consequences. Proper maintenance strategies and execution are thus necessary [1-2] to keep a component in functional state and limit the risk and costs associated to its failure. Many strategies can be considered, from corrective maintenance (CM) upon failure, to preventive maintenance (PM) in the form of scheduled repairs or replacements, to Condition-Based Maintenance (CBM) [3-4].

Mathematically, maintenance planning can be framed as a problem of decision under uncertainty. The resolution of the maintenance optimization problem thus implies the use of stochastic models [5]. The objective is generally to select maintenance parameters, such as the age-replacement interval or the preventive repair level, in order to minimize the expected total operation cost or system unavailability, given some prior knowledge on random degradation mechanisms affecting the component. Extensive literature exists in the domain of maintenance optimization and the possible resolution approaches, see e.g. [3-8].

It is intuitively recognized that gathering information that reduces uncertainty on a component's current state or on its degradation rate, maintenance decisions can be made that result in improved safety and cost-efficiency [2][4][7]. Additional information can be collected by inspection, either periodic, aperiodic or continuous, see e.g. [9-11].
The opportunity of information gathering and processing has driven up the interest in CBM policies and in the so-called Prognostics and Health Management (PHM) field [12-16]. In particular, a prognostic of the Remaining Useful Life (RUL) of a component appears intuitively as a valuable piece of information for decision-making.

However, a quantitative analysis of the mechanisms by which the uncertainty on the degradation state may be reduced and its effect on the health management decision process is not a question that has received strong attention. The notion of Value of Information (VoI), based on the seminal works in [1718], at the junction of Bayesian statistics and decision theory, can be a particularly relevant tool for that purpose. VoI may be used to quantify the value of collecting additional information, thus reducing uncertainty, in the context of an uncertain decision-making process. It has become quite popular in the Structural Health Monitoring (SHM) community, as a way to compare the merits of different, and often expensive, monitoring systems, see e.g. [19-22]. Recent works also consider VoI for sequential health management decisions [23].

The main objective of this paper is to develop the application of the concept of $\mathrm{VoI}$ in the framework of the health management of a component subject to degradation. While for most problems of maintenance planning, the goal is to come to an optimal policy, here one is interested in assessing the value of a given information gathering mechanism. In particular, it is proposed to analyze the action which consists in obtaining the current condition of a component whose degradation can be modeled by a gamma process.

The rest of this paper is organized as follows. Section II, describes the use of gamma process models for degradation and maintenance optimization. In Section III, the notion of VoI is introduced as a means to quantify the effect of uncertainty reduction. VoI calculation is applied in Section IV to a case where the current condition of a component undergoing a gamma degradation process is inspected. In Section V, a discussion is proposed on this particular information gathering choice in the context of PHM and maintenance optimization. 


\section{GAMMA DEGRADATION PROCESS AND MAINTENANCE OPTIMIZATION}

\section{A. Gamma process for degradation}

Due to inherent randomness, e.g. in material properties or load conditions, the time at which a given component fails is necessarily described by a random value. Various stochastic models can be considered to describe this randomness. They generally belong to one of the two following categories:

- Scalar representations, in the form of lifetime distribution models, such as exponential or Weibull distributions, and their connection with the so-called failure rate, see e.g. [5]; are well suited to characterize large populations of identical components:

- Continuous-time representations based on stochastic process theory; in this case, one is interested in accounting for the temporal variability of the condition of one specific individual, possibly in connection with the evolution of its operating environment. Generally, the failure of such individual component refers to the crossing of a threshold value, e.g. material strength. Continuous-time representations are more suited for Condition-Based Maintenance.

For continuous-time representations, Gamma processes have gained much interest in degradation modeling [8][24]. First, they are quite amenable to mathematical treatment and closed-form expressions can be derived analytically. Second, gamma processes are non-decreasing, which is an appropriate representation for many monotonous degradation mechanisms.

A gamma process is a stochastic process with independent, non-negative random increments, having a gamma distribution with identical shape parameter [8]. If $X(t)$ is the condition of the component at time $t$, the probability distribution of $X(t)$ is given by (1):

$$
f_{X(t)}(x)=G a(x \mid v(t), u)
$$

where $x \rightarrow \operatorname{Ga}(x)$ is the probability density function of the Gamma distribution with shape parameter $v(t)$ and scale parameter $u$. $X(t)$ has gamma-distributed independent increments:

$$
X(\tau)-X(t) \sim G a(v(\tau)-v(t), u)
$$

At any time $t$, expectation and variance can be obtained :

$$
\begin{gathered}
E[X(t)]=v(t) / u \\
\operatorname{Var}[X(t)]=v(t) / u^{2}
\end{gathered}
$$

If $v(t)=v t$, where $v>0$ is a constant, the associated Gamma process is said to be stationary. In that sense, it can be considered as a more elaborate representation, e.g one with temporal variability, than linear degradation models of the form $X(t)=$ $A t$, with $A$ a random value among a population of components.

When failure of the component may be described by the crossing of a given threshold $y$, a closed-form expression gives the first hitting time of level $y$, which can be seen directly as the failure distribution $F(t)$, according to [8]:

$$
\begin{gathered}
F(t)=\operatorname{Pr}[X(t) \geq y] \\
=\int_{x=y}^{\infty} f_{X(t)}(x) d x=\frac{\Gamma(v(t), y u)}{\Gamma(v(t))}
\end{gathered}
$$

where $\Gamma(a, x)=\int_{z=x}^{\infty} z^{a-1} e^{-z} d z$ is the incomplete gamma function for $x \geq 0$ and $a>0$ and $\Gamma(x)=\int_{z=0}^{\infty} z^{x-1} e^{-z} d z$ is the gamma function for $x \geq 0$.

\section{B. Maintenance optimization and decision context}

Determining cost-efficient policies, either through preventive maintenance (PM) involving age-based repairs or replacement, or through condition-based maintenance (CBM) relying on monitoring or inspection, requires adequate stochastic models and resolution approaches. Given stochastic information on the degradation process, and decision-specific information on the costs of failure, replacement, repair, inspection or unavailability, the objective is to achieve minimal cost or unavailability, see e.g. [6-7]. Simply put, one wants to balance the cost of failures against the cost of repairs or replacements, generally on an infinite time horizon.

Resolution of the maintenance optimization problem can be based on exact methods and the use of renewal theory [5] or on numerical methods, see e.g. [7]. An extensive review of maintenance planning with gamma process models is provided by [8]. The needed information on the degradation usually lies within the failure distribution, $F(t)$, given by (5) for a gamma process, and a fixed threshold level $y$.

Let us propose a slightly different formulation from usual practice in maintenance optimization and introduce a framework inspired from decision theory. Let $L$ be a loss function representing total operation cost on a given time interval. The following decision context can be considered:

$$
L(\theta(t), t, a)=c_{F}(1-\theta(t))(1-a)+a c_{R}+\frac{c_{u}}{t}
$$

where $c_{F}$ is the cost of failure, $c_{R}$ is the cost of perfect repairs or replacement, $c_{u}$ describes the cost of unavailability, $\theta(t)$ is the state of the system on $[0, t[, t>0$, either functioning $(\theta=1)$ or failed $(\theta=0)$, and $a$ is an action or policy on [0, $t$, where replacement of the component is decided when $a=1$. If the system fails in $\left[0, t\right.$ [ and no repairs are carried out, cost $C_{F}$ is paid. If $\operatorname{cost} C_{R}$ is paid, no failure is possible on $[0, t[$. Unavailability cost decreases with $t$, as lifespan gets longer, assuming repair duration is small on a given cycle.

For simplicity of notation, $\theta$ will be used instead of $\theta(t)$. With the stochastic description of the degradation mechanism in (5), a probability measure can be assigned, to the state of the system $\theta$ on $[0, t[$ :

$$
\operatorname{Pr}[\theta=0]=\operatorname{Pr}[\exists u \in[0, t[, X(u)>y]=F(t)
$$

In the simplified framework above (6-7), the selection of a maintenance policy on $[0, t[$, resumes to the selection of $a$. Moreover, with perfect repair or replacement, $[0, t[$ can be considered a renewal cycle, see e.g. [5], and one can study the optimal policy, namely $a$, with different values of $t$. 


\section{VALUE OF INFORMATION (VOI)}

As described in [19], "the value of a piece of information depends on its ability to guide our decision". The concept of Value of Information (VoI), based on the seminal works in [1718], is rooted both in Bayesian statistics and decision theory, and provides a rational approach to attach a value to a piece of information. It allows to quantify the interest of gathering additional information in order to reduce uncertainty in the context of a decision-making process.

In Structural Health Monitoring (SHM), it may be used to quantify the merits and compare different, often expensive, monitoring systems. Here, the objective is to use VoI in the context of maintenance planning for a continuously degrading component.

Formally, VoI is calculated as the difference in expected cost, or loss, between the outcome of the best decisions that may be taken with and without the collection of additional information.

$$
V o I=L^{*}(\varnothing)-E_{z}\left[L^{*}(z)\right]
$$

$L^{*}(\varnothing)$ represents the minimal expected loss, associated to the optimal decision, when no additional information is available. $L^{*}(z)$ represents the minimal expected loss when the information from observation $z$ is available, thus restricting the uncertainty originally associated to the system state, and potentially leading to a decision with a 'better' outcome, on average.

As one does not know beforehand what the observation is going to be, $L^{*}(z)$ has to be averaged over all possible observations $z$. This approach is, thus, often denoted as a preposterior analysis (see [18] for a detailed discussion).

Introducing the expression of probability measures, in the case where $\theta$ is a continuous random value, one has:

$$
\begin{gathered}
V o I=\min _{a}\left(\int L(\theta, a) f_{\Theta}(\theta) d \theta\right) \\
-\int\left[\min _{a}\left(\int L(\theta \mid z, a) f_{\Theta \mid Z}(\theta \mid z) d \theta \mid z\right)\right] f_{Z}(z) d z
\end{gathered}
$$

where $f_{\Theta}(\theta)$ is the prior knowledge on the uncertainty relative to the system's state $\theta ; f_{\Theta \mid Z}(\theta \mid z)$ is the conditional measure which describes the reduction in uncertainty on $\theta$ when observation $z$ has been collected; $f_{Z}(z)$ is the measure over the possible observations, which ultimately depends on the system state uncertainty through the relation $f_{Z}(z)=$ $\int f_{Z \mid \Theta}(z \mid \theta) f_{\Theta}(\theta) d \theta$.

Let us insist on the fact that it is seen, through (9), that the VoI metric depends on three factors, namely: the decision context $L$ and possible actions $a$, the uncertainty originally associated to the system state, described by prior knowledge $f_{\Theta}(\theta)$, and the observation mechanism by which uncertainty may be reduced $f_{Z \mid \Theta}(z \mid \theta)$. The specification of all these factors are imperative for any VoI computation and necessarily make the result application-dependent.

\section{INSPECTION OF THE CURRENT CONDITION OF A COMPONENT}

In the context of a continuous and uncertain degradation process, being able to know the condition of the considered component, at a given time instance, is valuable from the point of view of maintenance decisions. VoI can quantify the potential gains from such an inspection.

Let us assume that the current condition $z$ of a component is inspected at time $\tau$. Given the knowledge of the degradation process in (5), the decision context defined in (6) and the relation introduced in (9) for the computation of $\mathrm{VoI}$, after simplification (here $\theta$ is a binary variable) one gets:

$$
\begin{gathered}
\operatorname{VoI}(t, \tau)=\min \left(c_{F} F(t), c_{R}\right) \\
-\int\left[\min \left(c_{F} F_{\mid z(\tau)}(t-\tau, z), c_{R}\right)\right] f_{Z(\tau)}(z) d z
\end{gathered}
$$

where $F(t)$ is the prior (unconditional) failure distribution for a gamma process given in $(5), f_{Z(\tau)}(z)$ is the gamma probability density function corresponding to the degradation process at time $\tau$, i.e. the distribution of $z \sim X(\tau)$, and $F_{\mid z(\tau)}(t-\tau, z)$ is the posterior (conditional) failure distribution given $z$ collected at time $\tau$. The latter can be obtained using (11), assuming that the considered gamma process is stationary, i.e. $v(t)=v t$, $v>0$.

$$
F_{\mid z(\tau)}(t-\tau, z)=\frac{\Gamma(v \times(t-\tau), u \times(y-z))}{\Gamma(v \times(t-\tau))}
$$

This last conditional density can be seen as the probability that the degradation increase in the interval $t-\tau$ exceeds the remaining capacity (or resistance) of the component $y-z$.

The interest of selecting a degradation model in the form of a stationary gamma process is that a closed-form expression can be given for both the conditional and unconditional distributions, and VoI can be computed easily. As it is the result of a pre-posterior analysis, $\operatorname{VoI}(t, \tau)$ is not dependent on any particular observation $z$. Here, the dependency on the decision context is seen through $t$, whereas the dependency on the information gathering process lies in the time instant $\tau$ when the inspection is realized.

An example of the simulation of degradation trajectories conditioned on one particular observation is given on Fig. 1. The set of parameter values chosen for the subsequent computation of $\mathrm{VoI}$ is given in TABLE I.

The first three parameters in TABLE I. characterize the degradation process and failure mechanism. They could be estimated in practice, e.g. using maximum likelihood estimation and (5), based on a sample of times to failure gathered from a population of similar components undergoing the same degradation process. The last three parameters specify the maintenance decision context, with associated costs for failure, repair/replacement and unavailability on a given cycle. 


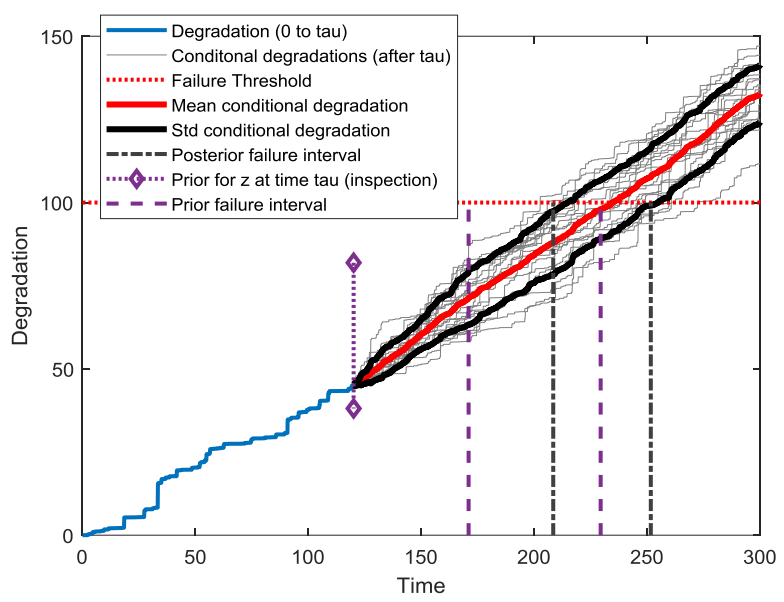

Fig. 1. Degradation, inspection and reduced uncertainty

TABLE I. PARAMETERS FOR VOI COMPUTATION EXAMPLE

\begin{tabular}{ccc} 
Parameter & Notation & Value \\
\hline Degradation rate & $v$ & 0.25 \\
Degradation variability & $u$ & 2 \\
Threshold & $y$ & 100 \\
Failure cost & $c_{F}$ & 300 \\
Repair/Replacement cost & $c_{R}$ & 50 \\
Unavailability cost on $[0, t[$ & $c_{u}$ & 300
\end{tabular}

In Fig. 1, the end value $z(\tau)$ for the first part of the trajectory generated, falls within the lower end of the prior distribution at time $\tau$. Consequently, the posterior distribution, in dash-dot line, tends to move to the right, with respect to the prior distribution, in dashed line. This particular observation $z(\tau)$ seems to indicate that for that component, it would be better to repair later than the usual, as its Remaining Useful Life (RUL) at $\tau$ seems to be higher than average. Let us also note that, as expected, the width of the posterior distribution is lower, since uncertainty has been reduced through the information in $z(\tau)$. For the computation of VoI, this individual result is averaged over all possible values for the observation $z(\tau)$, in order to assess the potential gains associated to an inspection at time $\tau$.

\section{RESULTS AND DISCUSSION}

\section{A. VoI computation and maintenance planning}

The result of $\mathrm{VoI}$ computation with parameter values in TABLE I. and using (10), is displayed in Fig. 2.

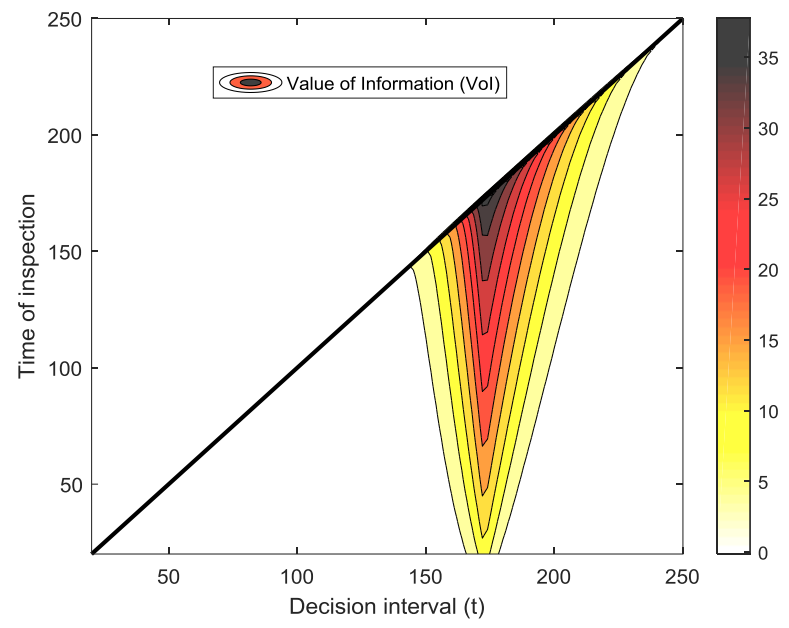

Fig. 2. Value of Information for one inspection at time $\tau$ and a decision context relative to $[0, t[$, e.g. a cycle's length.

Let us note that, VoI appears to be highest at a value close to $t=175$, which is actually close to the point where the prior optimal decision switches from 'do nothing', with average outcome $c_{F} F(t)$ to 'repair/replace' with outcome $c_{R}$, and those values become equal.

To illustrate, let us take for example an inspection at time $\tau=100$. When the component is operated for only a short life (but larger than $t=100$ ), the inspection will be of little value, because very few components will risk failure. Then, as one considers longer lives, it becomes interesting to inspect and $\mathrm{VoI}$ increases. When long lives are considered, VoI decreases both due to the fact that an inspection that is too early does not reduce uncertainty significantly and due to the increased need to repair/replace as time passes, regardless of what is the result of the inspection.

In Fig. 3, the results of the outcomes of decisions taken with and without additional information are compared. It is assumed here that information is collected at the most valuable time, which in this case is, 'in theory', 'just before the out uncertainty on $\theta$ unfolds at time $t^{\prime}$, thus $\tau=t^{-}$. This situation could be denoted as possessing 'perfect information'. This point will be detailed more clearly in section V.B.

Here one may notice that, for the optimal policy, the collection of additional information yields, on average, a small increase in component life, thus reducing unavailability cost on the considered cycle, and a small decrease of total operation cost associated to that cycle length (around $t=150$ ). For a practical application, that gain would have to be compared with the cost relative to the collection of information, through inspection. This last concern, although important in practice, is not the main 
object of the discussion here, where the interest is rather in the evolution of VoI on the time axis defined by $t$ and $\tau$.

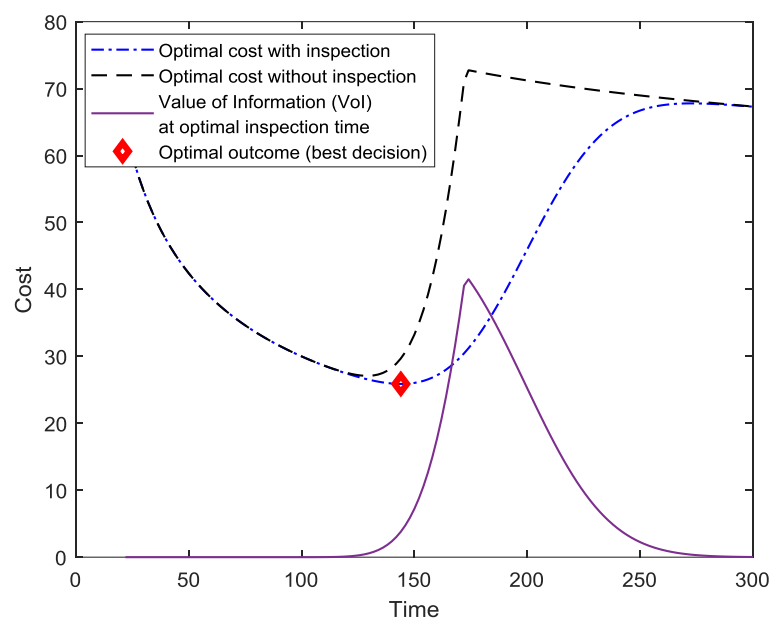

Fig. 3. VoI and alternative settings with and without information

It is seen that VoI is not dependent on unavailability $\operatorname{cost} c_{u}$, as the latter depends only on $t$ and not on $\theta$. It is also seen that the maximum of VoI does not necessarily correspond to the optimal decision with this particular information gathering mechanism and in this decision context.

\section{B. VoI in the context of late inspection}

The computation of VoI, in the decision context defined above, implies that once an observation has been taken, the decision to repair/replace the component can be made. This is not necessarily true in practice. Indeed, if the component is failed at inspection time, it is obviously too late to say that it has not failed in $[0, t[$, regardless of the selected action.

To account for that fact would require to define a more complicated decision problem wherein the action to inspect itself is not necessarily optimal, depending on the inspection time $\tau$. It is nonetheless possible to circumvent this problem, while retaining the same calculation framework, by assuming that the conditional outcome in (10) applies only to non-failed observations, and that the ones that would be failed are repaired before inspection (even if it is only possible in calculation and not in practice). In such case the expression $c_{F} F_{\mid z(\tau)}(t-\tau, z)$ is replaced by $c_{F} F_{\mid z(\tau)}(t-\tau, z)(1-F(\tau))$, thus conditioned on the component still being functional at inspection time. The result of this calculation is given in Fig 4.

In this latter figure it is seen that an inspection too close to the end of the interval, $\tau \approx t$, is in fact not that valuable, as a component already failed at inspection time $\tau$ will have logically failed on $[0, t$. Also, one may notice that no inspection is valuable past the point of prior decision switching $c_{F} F(t)=$ $c_{R} \approx 175$, as too much inspections will yield already failed components. In that latter case, it is preferable to repair/replace before $\tau$ rather than wait and inspect. This constitutes a specificity of the use of $\mathrm{VoI}$ in the context of time-related decision-making, necessarily appearing in PHM and CBM, in which the outcome of an observation can in fact restrict the action space in practice. More attention will be given to this issue in the authors' future works.

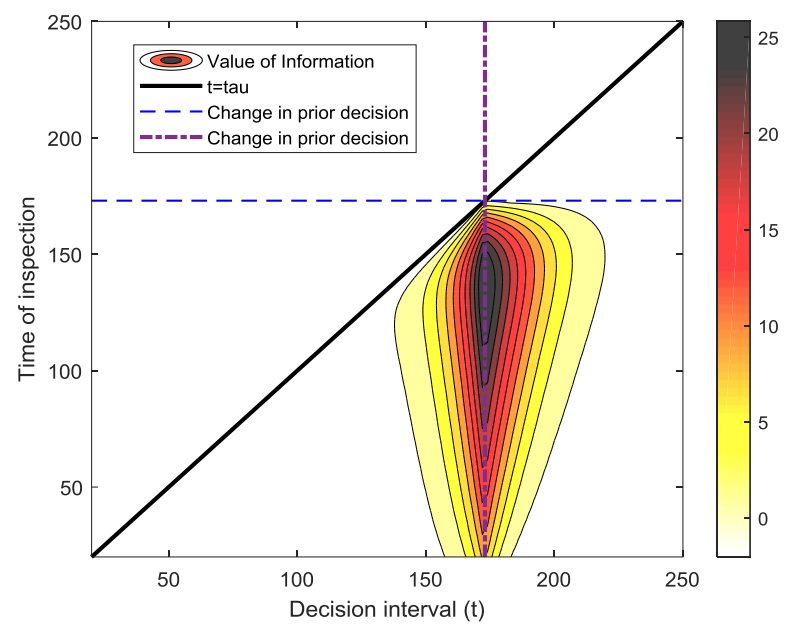

Fig. 4. Value of Information for one inspection at time $\tau$, accounting for late inspection issue

\section{CONCLUSION}

In this paper, the notion of Value of Information (VoI) has been discussed in the framework of Condition-Based Maintenance (CBM) strategies and in connection with an uncertain decision context based on a continuously degrading component. VoI is used to quantify the interest in collecting additional information, through inspection, regarding this continuous time degradation, for the purpose of reducing uncertainty on the outcome of health management decisions.

A closed-form expression for VoI computation has been derived for a gamma process and a situation where the action space is restricted to a 'do nothing (yet)' / 'replace' alternative. The cartography of VoI for different inspection times and component lifespans (or renewal cycle length), may help planning inspections, and ultimately save on operation cost or increase component's life.

Performing one inspection on a given cycle is a possible solution to gather additional information, but other solutions may be considered also, e.g. estimate the degradation rate which may be distributed within a population of components. In this sense, the use of VoI may be relevant to compare different information gathering mechanisms or strategies, in the context of PHM/CBM. It may be expected that a mechanism yielding more reduction in uncertainty generally leads to better average outcome, and VoI may be used to investigate various uncertainty reduction solutions.

Future works will include discussions of the different alternatives for the 'observation model' allowing to reduce uncertainty. Also one possible way to reduce uncertainty is through the use of prognostics models (PHM), which precision may have been evaluated beforehand, and VoI can be used as a metric to compare different prediction tools for $\mathrm{CBM}$ applications. 


\section{ACKNOWLEDGMENT}

The authors acknowledge the support of the Chair System Science and the Energy Challenge, Fondation Electricité de France (EDF).

\section{REFERENCES}

[1] Dekker, R. (1996). Applications of maintenance optimization models: a review and analysis. Reliability engineering \& system safety, 51(3), 229240.

[2] Frangopol, D. M. (2011). Life-cycle performance, management, and optimisation of structural systems under uncertainty: accomplishments and challenges 1. Structure and Infrastructure Engineering, 7(6), 389-413.

[3] Wang, H. (2002). A survey of maintenance policies of deteriorating systems. European journal of operational research, 139(3), 469-489.

[4] Grall, A., Bérenguer, C., \& Dieulle, L. (2002). A condition-based maintenance policy for stochastically deteriorating systems. Reliability Engineering \& System Safety, 76(2), 167-180.

[5] Barlow, R. E., \& Proschan, F. (1996). Mathematical theory of reliability (Vol. 17). Siam.

[6] Zio, E., \& Compare, M. (2013). Evaluating maintenance policies by quantitative modeling and analysis. Reliability Engineering \& System Safety, 109, 53-65.

[7] Marseguerra, M., Zio, E., \& Podofillini, L. (2002). Condition-based maintenance optimization by means of genetic algorithms and Monte Carlo simulation. Reliability Engineering \& System Safety, 77(2), 151165 .

[8] Van Noortwijk, J. M. (2009). A survey of the application of gamma processes in maintenance. Reliability Engineering \& System Safety, 94(1), 2-21.

[9] Kallen, M. J., \& van Noortwijk, J. M. (2005). Optimal maintenance decisions under imperfect inspection. Reliability engineering \& system safety, 90(2-3), 177-185.

[10] Crowder, M., \& Lawless, J. (2007). On a scheme for predictive maintenance. European Journal of Operational Research, 176(3), 17131722 .
[11] Castanier, B., Grall, A., \& Bérenguer, C. (2005). A condition-based maintenance policy with non-periodic inspections for a two-unit series system. Reliability Engineering \& System Safety, 87(1), 109-120.

[12] Zio, E. (2016). Some challenges and opportunities in reliability engineering. IEEE Transactions on Reliability, 65(4), 1769-1782.

[13] Jardine, A. K., Lin, D., \& Banjevic, D. (2006). A review on machinery diagnostics and prognostics implementing condition-based maintenance. Mechanical systems and signal processing, 20(7), 1483-1510.

[14] Si, X. S., Wang, W., Hu, C. H., \& Zhou, D. H. (2011). Remaining useful life estimation-a review on the statistical data driven approaches. European journal of operational research, 213(1), 1-14.

[15] Lei, Y., Li, N., Guo, L., Li, N., Yan, T., \& Lin, J. (2018). Machinery health prognostics: A systematic review from data acquisition to RUL prediction. Mechanical Systems and Signal Processing, 104, 799-834.

[16] Heng, A., Zhang, S., Tan, A. C., \& Mathew, J. (2009). Rotating machinery prognostics: State of the art, challenges and opportunities. Mechanical systems and signal processing, 23(3), 724-739.

[17] Howard, R. A. (1966). Information value theory. IEEE Transactions on systems science and cybernetics, 2(1), 22-26.

[18] Raiffa, H. (1974). Applied statistical decision theory.

[19] Pozzi, M., \& Der Kiureghian, A. (2011, April). Assessing the value of information for long-term structural health monitoring. In Health monitoring of structural and biological systems 2011 (Vol. 7984, p. 79842W). International Society for Optics and Photonics.

[20] Zonta, D., Glisic, B., \& Adriaenssens, S. (2014). Value of information: impact of monitoring on decision - making. Structural Control and Health Monitoring, 21(7), 1043-1056.

[21] Straub, D. (2014). Value of information analysis with structural reliability methods. Structural Safety, 49, 75-85.

[22] Straub, D., Chatzi, E., Bismut, E., Courage, W., Döhler, M., Faber, M. H., ... \& Thöns, S. (2017, August). Value of information: A roadmap to quantifying the benefit of structural health monitoring. In ICOSSAR-12th International Conference on Structural Safety \& Reliability.

[23] Memarzadeh, M., \& Pozzi, M. (2016). Value of information in sequential decision making: Component inspection, permanent monitoring and system-level scheduling. Reliability Engineering \& System Safety, 154, 137-151.

[24] Abdel-Hameed, M. (1975). A gamma wear process. IEEE transactions on Reliability, 24(2), 152-153. 